\title{
WPO, COV and IIA bargaining solutions
}

\author{
Citation for published version (APA):
}

Peters, H. J. M., \& Vermeulen, A. J. (2006). WPO, COV and IIA bargaining solutions. METEOR, Maastricht University School of Business and Economics. METEOR Research Memorandum No. 021 https://doi.org/10.26481/umamet.2006021

Document status and date:

Published: 01/01/2006

DOI:

10.26481/umamet.2006021

Document Version:

Publisher's PDF, also known as Version of record

\section{Please check the document version of this publication:}

- A submitted manuscript is the version of the article upon submission and before peer-review. There can be important differences between the submitted version and the official published version of record.

People interested in the research are advised to contact the author for the final version of the publication, or visit the DOI to the publisher's website.

- The final author version and the galley proof are versions of the publication after peer review.

- The final published version features the final layout of the paper including the volume, issue and page numbers.

Link to publication

\footnotetext{
General rights rights.

- You may freely distribute the URL identifying the publication in the public portal. please follow below link for the End User Agreement:

www.umlib.nl/taverne-license

Take down policy

If you believe that this document breaches copyright please contact us at:

repository@maastrichtuniversity.nl

providing details and we will investigate your claim.
}

Copyright and moral rights for the publications made accessible in the public portal are retained by the authors and/or other copyright owners and it is a condition of accessing publications that users recognise and abide by the legal requirements associated with these

- Users may download and print one copy of any publication from the public portal for the purpose of private study or research.

- You may not further distribute the material or use it for any profit-making activity or commercial gain

If the publication is distributed under the terms of Article $25 \mathrm{fa}$ of the Dutch Copyright Act, indicated by the "Taverne" license above, 
Hans Peters, Dries Vermeulen

WPO, COV and IIA bargaining solutions

RM/06/021

JEL code: C72, D44

\section{METE@R}

Maastricht research school of Economics

of TEchnology and ORganizations

Universiteit Maastricht

Faculty of Economics and Business Administration P.O. Box 616

NL - 6200 MD Maastricht

phone : ++31433883830

fax : ++31433884873 



\title{
WPO, COV and IIA bargaining solutions
}

\author{
Hans Peters * Dries Vermeulen ${ }^{\dagger}$
}

June 2006

\begin{abstract}
The class of bargaining solutions that are defined on the domain of finite sets of alternatives and satisfy Weak Pareto Optimality (WPO), Independence of Irrelevant Alternatives (IIA) and Covariance (COV), is characterized. These solutions select from the set of maximizers of a nonsymmetric Nash product - i.e., from a nonsymmetric (multi-valued) Nash bargaining solution - according to a specific decomposition of the indifference curves of this Nash product. We use this characterization in two ways. First, we derive consequences on this domain and on larger domains of compact (non-convex) bargaining problems, and show that most results in the literature are special cases and consequences of our central results - in particular by adding continuity or symmetry axioms. Second, since the continuity axiom prevents nontrivial selections from the Nash bargaining solutions, we use the Axiom of Choice to construct for example non-single-valued discontinuous WPO, IIA and COV bargaining solutions. It is conjectured that, in the case of two-person bargaining problems, the existence of such discontinuous bargaining solutions cannot be shown from the Zermelo-Fraenkel axioms for set theory without using the Axiom of Choice.
\end{abstract}

JEL Codes. C72, D44.

Keywords. Nash bargaining solutions, non-convex bargaining problems, axiomatic characterization

\section{Introduction}

In his classical paper [8], Nash characterized the two-person Nash bargaining solution on the domain of convex bargaining problems by four axioms: weak Pareto optimality (WPO), symmetry (SYM), (scale) covariance (COV), and independence of irrelevant alternatives (IIA). Nash's paper has generated a large literature on different axiomatizations, different solutions and their axiomatizations, and different domains.

In the present paper we drop the convexity assumption and - for the larger part of the paper - consider finite $n$-person bargaining problems. We study multi-valued bargaining solutions

\footnotetext{
${ }^{*}$ h.peters@ke.unimaas.nl. Department of Quantitative Economics, Universiteit Maastricht, P.O. Box 616, 6200 MD Maastricht, The Netherlands.

${ }^{\dagger}$ d.vermeulen@ke.unimaas.nl. Department of Quantitative Economics, Universiteit Maastricht, P.O. Box 616, 6200 MD Maastricht, The Netherlands.
} 
satisfying WPO, IIA, and COV. The first two conditions retain their usual interpretation. WPO is a normative condition: an outcome should not be considered a good compromise if all bargainers can improve on it. IIA is a consistent choice condition: an outcome that is considered a best compromise should be considered a best compromise also within a smaller set. COV, however, should be interpreted with some care, since its usual justification based on expected utility is somewhat at odds with the lack of convexity. Here, we just assume that the preferences of the bargainers are represented uniquely up to choice of scale.

Before going into more details we note, first, that practically everything that is known thus far in the relevant literature follows from our characterization of this class of solutions: see below and see Section 8.3 for a comprehensive overview. Second, not including SYM or continuity beforehand opens the possibility of making nontrivial selections from the characterized multivalued (Nash) bargaining solutions.

The first main result of the paper (Theorem 3.3 and Corollary 3.4) is that an $n$-person bargaining solution satisfies these three axioms on the domain of all finite problems if and only if there is a nonnegative $n$-dimensional weight vector such that the solution selects a subset of the set of maximizers of the associated generalized Nash product. The second main result (Theorems 4.1 and 4.2) characterizes these selections in a precise manner: from each pair of Nash product maximizers, it tells us which element(s) is (are) chosen by the solution. Formally, this is described by a specific decomposition of the set of Nash product maximizers viewed as an Abelian multiplicative group.

These results are quite basic and have a large number of applications, both in the domain of finite bargaining problems and in larger domains. In the domain of finite problems, adding a continuity condition ( $\mathrm{CON}$ ) almost immediately implies the special case where all feasible maximizers of the Nash product are chosen by the solution: such solutions are called Nash solutions (Theorem 5.1). Thus, CON excludes any specific refinement of a Nash solution. This, and the fact that $\mathrm{CON}$ is a mathematical condition without very strong normative of behavioral appeal, are important reasons not to impose it from the outset. Adding SYM (a symmetry condition) has a similar effect: it implies that the solution is the symmetric $n$-person Nash bargaining solution, hence, it chooses all maximizers of the symmetric Nash product (Theorem 5.2). This practically implies a result by Mariotti [5], who characterizes the two-person symmetric Nash bargaining solution on the domain of finite bargaining problems.

Still in the finite case, we show in Section 5.2 that there is a plethora of discontinuous solutions 
that can be constructed by using the Axiom of Choice (or Zorn's Lemma). This is considered in detail for the two-player case in Section 6. We cannot prove but conjecture that the existence of non-single-valued discontinuous WPO, COV and IIA bargaining solutions cannot be derived without the Axiom of Choice.

In Section 7 we consider the consequences of our main results for bargaining solutions defined on larger domains of compact bargaining problems, containing the domain of finite problems. The latter condition is less restrictive than it might seem, since it would also be sufficient to assume that the comprehensive hulls of finite sets are in the domain, if one wants to exclude finite or disconnected sets. Since most of the existing literature is on compact and often comprehensive bargaining problems, this enables a comparison with results on Nash-like bargaining solutions defined on non-convex sets.

Our first result here is the observation that on any compact domain containing the finite domain, the conditions of WPO, IIA, COV, and CON again characterize the family of (nonsymmetric) Nash bargaining solutions (Theorem 7.1). This implies Kaneko's [4] characterization of the symmetric Nash bargaining correspondence as a special case. By adding SYM instead of CON, we obtain a straightforward generalization of Theorem 5.2, namely a characterization of the symmetric Nash bargaining solution on any compact domain containing the finite domain (Theorem 7.2). This result was recently obtained also by $\mathrm{Xu}$ and Yoshihara [11].

Further results are stated for the domain of all compact bargaining problems. This is for convenience: it is easy to see that smaller domains can be assumed as well. We show that on this domain specific discontinuous bargaining solutions are excluded (Theorem 7.3). A surprising consequence is that in the two-person case, dropping continuity adds only singlevalued solutions, namely those that either always pick the most left or always the most right point from the (nonsymmetric) Nash bargaining solution (Theorem 7.4). This result sheds special light on the main result in Zhou [12], which states that under a similar set of conditions plus single-valuedness, a solution selects from the maximizers of a Nash product. Theorem 7.4 shows that, at least in the two-person case, such single-valued solutions are limited to be of a very special kind. We conjecture that a similar restriction may hold in the $n$-person case but we do not yet have a complete characterization.

Some loose ends are tied in Section 8, which also includes a brief discussion of further related literature. Some auxiliary lemmas are collected in an appendix. 


\section{Preliminaries}

For $x, y \in \mathbb{R}^{n}, x \leq y$ means that $x_{i} \leq y_{i}$ for all $i$, and $x<y$ means that $x_{i}<y_{i}$ for all $i$. The vector in $\mathbb{R}^{n}$ whose coordinates are all zero is denoted by $0 . \mathbb{R}_{+}^{n}$ denotes the set of vectors $x$ in $\mathbb{R}^{n}$ with $x \geq 0$ and $\mathbb{R}_{++}^{n}$ denotes the set of vectors $x$ in $\mathbb{R}^{n}$ with $x>0$. For $a, x \in \mathbb{R}_{++}^{n}$, $a x:=\left(a_{1} x_{1}, \ldots, a_{n} x_{n}\right)$ and $\frac{x}{a}:=\left(\frac{x_{1}}{a_{1}}, \ldots, \frac{x_{n}}{a_{n}}\right)$. For $a \in \mathbb{R}^{n}$ and $S \subset \mathbb{R}^{n}, a S:=\{a x \mid x \in S\}$. We use the notation $S \subset T$ to indicate that $S$ is a subset of $T$, so $S=T$ is allowed.

A bargaining problem is a closed and non-empty set $B \subset \mathbb{R}_{++}^{n}$. (We implicitly assume the disagreement point to be the origin.) A non-empty set $\mathcal{D}$ of bargaining problems is called a domain. In this paper we mainly consider the domain

$$
\mathcal{F}:=\left\{F \subset \mathbb{R}_{++}^{n} \mid F \text { is finite and not empty }\right\}
$$

of all finite $n$-person bargaining problems. All other domains we consider are subsets of the domain $\mathcal{C}$ of all compact bargaining $n$-person bargaining problems.

A correspondence $\varphi: \mathcal{D} \rightarrow \mathbb{R}^{n}$ is called a (bargaining) solution when $\phi \neq \varphi(B) \subset B$ holds for all $B \in \mathcal{D}$. When specific elements, say $x$ and $y$ or $x^{1}, x^{2}$ and $x^{3}$ of $\mathbb{R}_{++}^{n}$ are involved, we typically write $\varphi(x, y)$ and $\varphi\left(x^{1}, x^{2}, x^{3}\right)$ instead of $\varphi(\{x, y\})$ and $\varphi\left(\left\{x^{1}, x^{2}, x^{3}\right\}\right)$.

We define several properties that a bargaining solution $\varphi$ may (or may not) satisfy.

PO (Pareto Optimality)

For every $B \in \mathcal{D}$ and all $x, y \in B, x \leq y$ and $x \neq y$ implies that $x \notin \varphi(B)$.

WPO (Weak Pareto Optimality)

For every $B \in \mathcal{D}$ and all $x, y \in B, x<y$ implies that $x \notin \varphi(B)$.

IIA (Independence of Irrelevant Alternatives)

For all $B, C \in \mathcal{D}$ with $C \subset B$ and $C \cap \varphi(B) \neq \phi$ we have $\varphi(C)=C \cap \varphi(B)$.

COV (Covariance)

For all $a \in \mathbb{R}_{++}^{n}$ and all $B \in \mathcal{D}$ such that $a B \in \mathcal{D}, \varphi(a B)=a \varphi(B)$.

CON (Continuity)

Let $x^{k}, y^{k} \in \mathbb{R}_{++}^{n}$ for all $k \in \mathbb{N}, x^{k} \rightarrow x \in \mathbb{R}_{++}^{n}$ and $y^{k} \rightarrow y \in \mathbb{R}_{++}^{n}$. If $\left\{x^{k}, y^{k}\right\},\{x, y\} \in \mathcal{D}$ and $x^{k} \in \varphi\left(x^{k}, y^{k}\right)$ for all $k \in \mathbb{N}$, then $x \in \varphi(x, y)$.

SYM (Symmetry)

Let $B \in \mathcal{D}$ be symmetric, i.e., $B$ is invariant under any permutation of coordinates. If $x \in \varphi(B)$ and $y \in B$ can be obtained from $x$ by permuting its coordinates, then $y \in \varphi(B)$. 
The properties of WPO, IIA, COV, and SYM were introduced by Nash [8] for single-valued solutions. ${ }^{1}$

Let $\mathcal{D}$ be a domain with $\mathcal{F} \subset \mathcal{D}$. A solution $\varphi: \mathcal{D} \rightarrow \mathbb{R}_{++}^{n}$ is called transitive if, for all $x_{1}, x_{2}, x_{3} \in \mathbb{R}_{++}^{n}$, from $x_{2} \in \varphi\left(x_{1}, x_{2}\right)$ and $x_{3} \in \varphi\left(x_{2}, x_{3}\right)$ it follows that $x_{3} \in \varphi\left(x_{1}, x_{3}\right)$. We shall frequently use the following observation.

Lemma 2.1 Any solution $\varphi: \mathcal{D} \rightarrow \mathbb{R}_{++}^{n}$ that satisfies IIA is transitive.

Proof. Suppose that $\varphi$ satisfies IIA, that $x_{2} \in \varphi\left(x_{1}, x_{2}\right)$, and that $x_{3} \in \varphi\left(x_{2}, x_{3}\right)$. Suppose that $\varphi\left(x_{1}, x_{2}, x_{3}\right)$ does not contain $x_{3}$. Then, because $x_{3} \in \varphi\left(x_{2}, x_{3}\right)$, it does also not contain $x_{2}$ by IIA. Consequently, because $x_{2} \in \varphi\left(x_{1}, x_{2}\right)$, it does also not contain $x_{1}$ by IIA. This contradicts the assumption that $\varphi\left(x_{1}, x_{2}, x_{3}\right)$ is not empty. Hence, $\varphi\left(x_{1}, x_{2}, x_{3}\right)$ contains $x_{3}$.

A well-known class of bargaining solutions is the class of (asymmetric) Nash bargaining solutions. Let $\mathcal{D}$ be a domain with $\mathcal{D} \subset \mathcal{C}^{2}$. For a non-zero vector $\alpha=\left(\alpha_{i}\right)_{i \in N} \in \mathbb{R}_{+}^{n}$ the $\alpha$-Nash bargaining solution $N^{\alpha}$ is defined by, for all $B \in \mathcal{D}$,

$$
N^{\alpha}(B)=\left\{x \in B \mid \prod_{i} x_{i}^{\alpha_{i}} \geq \prod_{i} y_{i}^{\alpha_{i}} \text { for all } y \in B\right\} .
$$

A function of the form $x \mapsto \prod_{i} x_{i}^{\alpha_{i}}$ is called a Nash product. A solution $\varphi$ is called a Nash bargaining solution if there exists a vector $\alpha$ such that $\varphi=N^{\alpha}$. All Nash bargaining solutions satisfy WPO, IIA, COV and CON. If $\alpha \in \mathbb{R}_{++}^{n}$, then $N^{\alpha}$ even satisfies PO. Further, notice that $N^{\alpha}=N^{\lambda \alpha}$ for any positive real number $\lambda$. Thus we may assume w.l.o.g. that $\sum_{i} \alpha_{i}=1$.

\section{WPO, COV and IIA bargaining solutions}

In this and the next section we deal exclusively with the domain $\mathcal{F}$ of all finite bargaining problems. On this domain we study the set of all WPO, COV and IIA bargaining solutions. In this section we introduce the notion of a generalized indifference curve of a WPO, COV and IIA bargaining solution, and show that a generalized indifference curve necessarily coincides with the indifference curve of a Nash bargaining solution. Consequently, each WPO, COV and IIA bargaining solution is a refinement of a Nash bargaining solution.

Let $\varphi: \mathcal{F} \rightarrow \mathbb{R}_{++}^{n}$ be a bargaining solution that satisfies WPO, COV and IIA. Take $x \in \mathbb{R}_{++}^{n}$. Define for $y \in \mathbb{R}_{++}^{n}$,

$$
\lambda(x, y):=\inf \{\lambda>0 \mid \lambda y \in \varphi(x, \lambda y)\} .
$$

\footnotetext{
${ }^{1}$ For the IIA-axiom see Shubik [10], p. 421, who attributes it to an informal note of Nash.

${ }^{2}$ We merely use compactness to guarantee non-emptiness of the Nash bargaining solutions.
} 
By Lemma A.1 this is a sound definition and $\lambda(x, y)>0 .{ }^{3}$ Further define

$$
I_{\varphi}(x):=\left\{\lambda(x, y) y \mid y \in \mathbb{R}_{++}^{n}\right\}
$$

The set $I_{\varphi}(x)$ is a subset of $\mathbb{R}_{++}^{n}$. It is called the generalized indifference curve through $x$. We briefly discuss the reason for this name. As we will see, $I_{\varphi}(x)$ is indeed the indifference curve through $x$ whenever $\varphi$ satisfies $\mathrm{CON}$, in the sense that $I_{\varphi}(x)$ equals the set of points $y \in \mathbb{R}_{++}^{n}$ with $\varphi(x, y)=\{x, y\}$ in that case. However, in general we only have the following fact.

Lemma 3.1 For every $x, y \in \mathbb{R}_{++}^{n}$, if $\lambda<\lambda(x, y)$ then $\varphi(x, \lambda y)=\{x\}$, and if $\lambda>\lambda(x, y)$ then $\varphi(x, \lambda y)=\{\lambda y\}$

Proof. We only show the first statement. Let $\lambda<\lambda(x, y)$ and suppose that $\lambda y \in \varphi(x, \lambda y)$. Take a $\mu$ with $\lambda<\mu<\lambda(x, y)$. Notice that $\varphi(\lambda y, \mu y)=\{\mu y\}$ by WPO and the non-emptiness of $\varphi(\lambda y, \mu y)$. Thus, $\mu y \in \varphi(x, \mu y)$ by transitivity of $\varphi$, which contradicts the definition of $\lambda(x, y)$. Hence, $\lambda y \notin \varphi(x, \lambda y)$ and then $\varphi(x, \lambda y)=\{x\}$ by non-emptiness.

Thus, when $\varphi$ does not (necessarily) satisfy CON, the point $\lambda(x, y) y$ is exactly the point of the form $\lambda y$ on the ray emanating from the origin through $y$ where we switch from choosing $\varphi(x, \lambda y)=\{\lambda y\}$ when $\lambda>\lambda(x, y)$ to choosing $\varphi(x, \lambda y)=\{x\}$ when $\lambda<\lambda(x, y)$. The choice for $\varphi(x, \lambda(x, y) y)$ itself is left unspecified. ${ }^{4}$

We can say this somewhat differently. Notice that $I_{\varphi}(x)=\left\{y \in \mathbb{R}_{++}^{n} \mid \lambda(x, y)=1\right\}$. Thus $I_{\varphi}(x)$ is the set of points $y \in \mathbb{R}_{++}^{n}$ where we switch from choosing $\varphi(x, \lambda y)=\{\lambda y\}$ when $\lambda>1$ to choosing $\varphi(x, \lambda y)=\{x\}$ when $\lambda<1$. Again, the choice for $\varphi(x, y)$ itself is left unspecified. It can be either $\{x\}$, or $\{y\}$, or $\{x, y\}$ (although this choice cannot be made entirely arbitrarily as we will see in the next section).

We show that the generalized indifference curve $I_{\varphi}(x)$ is in fact the indifference curve of an $\alpha$-Nash bargaining solution for some $\alpha$. In order to show this, define $e \in \mathbb{R}_{++}^{n}$ by $e_{i}:=1$ for all $i$, and for $x \in \mathbb{R}_{++}^{n}$, define $\lambda(x):=\lambda(e, x)$. Next, define $a^{j} \in \mathbb{R}_{++}^{n}$ by

$$
a_{i}^{j}:= \begin{cases}\frac{1}{2} & \text { if } i=j \\ 1 & \text { else. }\end{cases}
$$

Define the vector $\alpha=\left(\alpha_{1}, \ldots, \alpha_{n}\right)$ by

$$
\alpha_{j}:={ }^{2} \log \lambda\left(a^{j}\right) .
$$

\footnotetext{
${ }^{3}$ In fact $\lambda(x, y)$ depends on the solution $\varphi$ and we should write for example $\lambda_{\varphi}(x, y)$. Usually, however, it is clear which solution $\varphi$ is meant and we simply write $\lambda(x, y)$.

${ }^{4}$ The construction bears similarity to the classical construction of a utility function representing a binary relation (e.g., [2]). Note, however, that we do not require continuity.
} 
Notice that $e \geq a^{j}$. Hence, $\lambda\left(a^{j}\right) \geq 1$, and therefore $\alpha_{j} \geq 0$. Moreover, we have the following lemma.

Lemma $3.2 \sum_{i} \alpha_{i}=1$.

Proof. We show $\prod_{j} \lambda\left(a^{j}\right)=2$ in two steps.

A. Take $j \in N$. Take $\lambda>\lambda\left(a^{j}\right)$. Then $\varphi\left(e, \lambda a^{j}\right)=\left\{\lambda a^{j}\right\}$ by Lemma 3.1. Define $d^{j} \in \mathbb{R}_{++}^{n}$ by

$$
d_{i}^{j}:= \begin{cases}1 & \text { if } i=j \\ \frac{1}{2} & \text { else. }\end{cases}
$$

Notice that $\left(\frac{2}{\lambda} d^{j}\right)\left(\lambda a^{j}\right)=e$. So, by COV,

$$
\varphi\left(\frac{2}{\lambda} d^{j}, e\right)=\varphi\left(\left(\frac{2}{\lambda} d^{j}\right) e,\left(\frac{2}{\lambda} d^{j}\right)\left(\lambda a^{j}\right)\right)=\left\{\left(\frac{2}{\lambda} d^{j}\right)\left(\lambda a^{j}\right)\right\}=\{e\} .
$$

So, $\frac{2}{\lambda} \leq \lambda\left(d^{j}\right)$ for all $\lambda>\lambda\left(a^{j}\right)$, and hence $2 \leq \lambda\left(d^{j}\right) \cdot \lambda\left(a^{j}\right)$. Conversely, take $\lambda<\lambda\left(a^{j}\right)$. Then $\varphi\left(e, \lambda a^{j}\right)=\{e\}$ by Lemma 3.1. So, by COV,

$$
\varphi\left(\frac{2}{\lambda} d^{j}, e\right)=\varphi\left(\left(\frac{2}{\lambda} d^{j}\right) e,\left(\frac{2}{\lambda} d^{j}\right)\left(\lambda a^{j}\right)\right)=\left\{\left(\frac{2}{\lambda} d^{j}\right) e\right\}=\left\{\frac{2}{\lambda} d^{j}\right\} .
$$

So, $\frac{2}{\lambda} \geq \lambda\left(d^{j}\right)$ for all $\lambda<\lambda\left(a^{j}\right)$, and thus $2 \geq \lambda\left(d^{j}\right) \cdot \lambda\left(a^{j}\right)$. Hence, $\lambda\left(d^{j}\right) \cdot \lambda\left(a^{j}\right)=2$.

B. Now notice that $d^{j}=\prod_{i \neq j} a^{i}$. Hence, we are done if we can show that $\lambda(x) \cdot \lambda(y)=\lambda(x y)$ for any $x, y \in \mathbb{R}_{++}^{n}$. Take $x, y \in \mathbb{R}_{++}^{n}$. Take $\lambda>\lambda(x)$ and $\mu>\lambda(y)$. Then $\varphi(\lambda x, e)=\{\lambda x\}$ and $\varphi(\mu y, e)=\{\mu y\}$ by Lemma 3.1. So, by COV and transitivity, $\varphi(\lambda \mu x y, e)=\{\lambda \mu x y\}$ and $\lambda \mu \geq \lambda(x y)$. Hence, $\lambda(x) \cdot \lambda(y) \geq \lambda(x y)$. Conversely, take $\lambda<\lambda(x)$ and $\mu<\lambda(y)$. Then $\varphi(\lambda x, e)=\{e\}$ and $\varphi(\mu y, e)=\{e\}$ by Lemma 3.1. So, by COV and transitivity, $\varphi(\lambda \mu x y, e)=$ $\{e\}$ and $\lambda \mu \leq \lambda(x y)$. Hence, $\lambda(x) \cdot \lambda(y) \leq \lambda(x y)$. Altogether $\lambda(x) \cdot \lambda(y)=\lambda(x y)$.

Now we can show the main result of this section, namely that the generalized indifference curve $I_{\varphi}(x)$ equals the indifference curve through $x$ of the $\alpha$-Nash bargaining solution.

Theorem 3.3 For all $x, y \in \mathbb{R}_{++}^{n}$ we have $y \in I_{\varphi}(x)$ if and only if

$$
\prod_{i} x_{i}^{\alpha_{i}}=\prod_{i} y_{i}^{\alpha_{i}}
$$

Proof. Define $H$ as the set of vectors $y \in \mathbb{R}_{++}^{n}$ with $\prod_{i} y_{i}^{\alpha_{i}}=1$. By COV it suffices to show that $I_{\varphi}(e)=H$. We prove this as follows. Write $b^{j}:=\lambda\left(a^{j}\right) a^{j}$. Let $B$ be the collection of vectors in $\mathbb{R}_{++}^{n}$ that can be written as

$$
\prod_{j=1}^{n}\left(b^{j}\right)^{\mu_{j}}
$$


with $\mu_{1}, \ldots, \mu_{n} \in \mathbb{R}$. First we show that $B=H$. Then we show that $B \subset I_{\varphi}(e)$. Finally we argue that $I_{\varphi}(e)=H$.

A1. Take $b=\prod_{j=1}^{n}\left(b^{j}\right)^{\mu_{j}} \in B$. Then

$$
\begin{aligned}
\prod_{i} b_{i}^{\alpha_{i}} & =\prod_{i}\left(\prod_{j}\left(b_{i}^{j}\right)^{\mu_{j}}\right)^{\alpha_{i}} \\
& =\prod_{i}\left(\prod_{j}\left(\lambda\left(a^{j}\right) \cdot a_{i}^{j}\right)^{\mu_{j}}\right)^{\alpha_{i}} \\
& =\prod_{i} \prod_{j}\left(\lambda\left(a^{j}\right) \cdot a_{i}^{j}\right)^{\alpha_{i} \mu_{j}} \\
& =\prod_{j} \prod_{i} \lambda\left(a^{j}\right)^{\alpha_{i} \mu_{j}} \cdot \prod_{i} \prod_{j}\left(a_{i}^{j}\right)^{\alpha_{i} \mu_{j}} \\
& =\prod_{j} \lambda\left(a^{j}\right)^{\mu_{j} \sum_{i} \alpha_{i}} \cdot \prod_{i}\left(a_{i}^{i}\right)^{\alpha_{i} \mu_{i}} \\
& =\prod_{j} \lambda\left(a^{j}\right)^{\mu_{j}} \cdot \prod_{i}\left(\frac{1}{2}\right)^{\alpha_{i} \mu_{i}} \\
& =\prod_{j} \lambda\left(a^{j}\right)^{\mu_{j}} \cdot \prod_{i}\left(\frac{1}{2}\right)^{\mu_{i} \cdot{ }^{2} \log \lambda\left(a^{i}\right)} \\
& =\prod_{j} \lambda\left(a^{j}\right)^{\mu_{j}} \cdot\left(\prod_{i} \lambda\left(a^{i}\right)^{\mu_{i}}\right)^{-1}=1,
\end{aligned}
$$

where the $5^{\text {th }}$ equality follows from the definition of $a^{j}$, while the $6^{\text {th }}$ equality follows from both the definition of $a^{j}$ and Lemma 3.2. Hence, $b \in H$.

A2. Conversely, let $y \in H$. So, $y \in \mathbb{R}_{++}^{n}$ and $\prod_{i} y_{i}^{\alpha_{i}}=1$. We show that there are $\mu_{1}, \ldots, \mu_{n} \in \mathbb{R}$ such that for all $i$

$$
y_{i}=\prod_{j=1}^{n}\left(b_{i}^{j}\right)^{\mu_{j}} .
$$

Write $z_{i}:={ }^{2} \log y_{i}$. Since $b_{i}^{j}=\lambda\left(a^{j}\right) a_{i}^{j}$ by definition, we have $b_{i}^{j}=\lambda\left(a^{j}\right)$ for all $j \neq i$ and $b_{i}^{i}=\frac{1}{2} \lambda\left(a^{i}\right)$. Thus, taking base 2 logarithms in (1) shows that we have to find $\mu_{1}, \ldots, \mu_{n} \in \mathbb{R}$ such that for all $i$

$$
z_{i}=-\mu_{i}+\sum_{j} \alpha_{j} \mu_{j}
$$

Write

$$
A=\left[\begin{array}{cccc}
\alpha_{1}-1 & \alpha_{2} & \cdots & \alpha_{n} \\
\alpha_{1} & \alpha_{2}-1 & \cdots & \alpha_{n} \\
\vdots & \vdots & \ddots & \vdots \\
\alpha_{1} & \alpha_{2} & \cdots & \alpha_{n}-1
\end{array}\right], \quad \mu=\left[\begin{array}{c}
\mu_{1} \\
\vdots \\
\mu_{n}
\end{array}\right] \quad \text { and } \quad z=\left[\begin{array}{c}
z_{1} \\
\vdots \\
z_{n}
\end{array}\right]
$$

We show that $z=A \mu$ has a solution. Let $Z$ be the set of vectors $x \in \mathbb{R}^{n}$ with $\sum_{i} \alpha_{i} x_{i}=0$. Since $\sum_{i} \alpha_{i}=1$, we have $\operatorname{dim}(Z)=n-1$. Further, from A1, each vector of the form $\prod_{j=1}^{n}\left(b^{j}\right)^{\mu_{j}}$ 
is an element of $H$. Taking base 2 logarithms this implies that each vector of the form $A \mu$ is an element of $Z$. Thus, $\operatorname{Im}(A)$ is a subspace of $Z$. However, subtracting the first row of $A$ from its other rows yields the matrix

$$
B=\left[\begin{array}{ccccc}
\alpha_{1}-1 & \alpha_{2} & \alpha_{3} & \cdots & \alpha_{n} \\
-1 & -1 & 0 & \cdots & 0 \\
-1 & 0 & -1 & \cdots & 0 \\
\vdots & \vdots & \vdots & \ddots & \vdots \\
-1 & 0 & 0 & \cdots & -1
\end{array}\right]
$$

So, $\operatorname{dim}(\operatorname{Im}(A))=\operatorname{rank}(A)=\operatorname{rank}(B) \geq n-1$, and $\operatorname{Im}(A)=Z$. However, since $\prod_{i} y_{i}^{\alpha_{i}}=1$, we have $\sum_{i} \alpha_{i} z_{i}=0$ and $z$ is an element of $Z$. Hence, the system $z=A \mu$ of linear equations has a solution.

B. We show that $B \subset I_{\varphi}(e)$. Take $q_{1}, \ldots, q_{n} \in \mathbb{Q}$. First we show that $\prod_{j=1}^{k} b(j)^{q_{j}} \in I_{\varphi}(e)$ for $k \leq n$. For $k=1$ this follows immediately from Lemma A.5. Now suppose that $\prod_{j=1}^{k} b(j)^{q_{j}} \in$ $I_{\varphi}(e)$. Then, by Lemmas A.2, A.4 and A.5, $\prod_{j=1}^{k+1} b(j)^{q_{j}} \in I_{\varphi}\left(b(k+1)^{q_{k+1}}\right)=I_{\varphi}(e)$. Hence, since $I_{\varphi}(e)$ is closed by Lemma A.3, we obtain $B \subset I_{\varphi}(e)$.

C. From $\mathrm{A}$ and $\mathrm{B}$ we conclude $H \subset I_{\varphi}(e)$. However, since each ray in $\mathbb{R}_{++}^{n}$ emanating from the origin clearly intersects $H$ exactly once, and also intersects $I_{\varphi}(e)$ exactly once by Lemma A.1, necessarily $I_{\varphi}(e)=H$.

Let $\varphi: \mathcal{F} \rightarrow \mathbb{R}_{++}^{n}$ and $\psi: \mathcal{F} \rightarrow \mathbb{R}_{++}^{n}$ be two bargaining solutions. We say that $\varphi$ is a refinement of $\psi$ when $\varphi(F) \subset \psi(F)$ for all $F \in \mathcal{F}$. We have:

Corollary 3.4 Any bargaining solution that satisfies WPO, IIA and COV is a refinement of a (uniquely determined) Nash bargaining solution.

Proof. Let $\varphi$ be a bargaining solution that satisfies WPO, IIA and COV. Let $\alpha=\left(\alpha_{1}, \ldots, \alpha_{n}\right)$ be as defined before. Take $F \in \mathcal{F}$ and $x \in \varphi(F)$. Suppose that $x \notin N^{\alpha}(F)$. Then there is a $y \in F$ with

$$
\prod_{i} x_{i}^{\alpha_{i}}<\prod_{i} y_{i}^{\alpha_{i}}
$$

Thus, $\lambda(x, y)<1$ by Theorem 3.3. Consequently $\varphi(x, y)=\{y\}$ by Lemma 3.1, which violates IIA. Hence, $x \in N^{\alpha}(F)$.

Thus we can view every WPO, IIA and COV bargaining solution as arising from a Nash bargaining solution, with the additional proviso that ties, or indifferences, within the indifference curve of the Nash bargaining solution may be broken one way or the other. How exactly ties can be broken is the topic of the next section. 


\section{LDR decomposition of the generalized indifference curve}

In this section we show that for a WPO, IIA and COV bargaining solution, ties within the indifference curve of the enveloping Nash bargaining solution can only be broken in a specific way, namely only in a way that respects the covariance requirement together with transitivity. We give a precise description of the consequences of this observation.

Let $\varphi: \mathcal{F} \rightarrow \mathbb{R}_{++}^{n}$ be a WPO, IIA and COV bargaining solution. Consider the generalized indifference curve $I_{\varphi}:=I_{\varphi}(e)$ through the unit vector $e$. Notice that $I_{\varphi}$ is an Abelian multiplicative group under the operation defined by $(x y)_{i}:=x_{i} \cdot y_{i}$. Indeed, if $x, y \in I_{\varphi}$, then $x y \in I_{\varphi}$ and $\frac{e}{x} \in I_{\varphi}$ by Theorem 3.3. Define the subsets $L_{\varphi}, D_{\varphi}$ and $R_{\varphi}$ of $I_{\varphi}$ by

$$
\begin{aligned}
L_{\varphi} & :=\left\{x \in I_{\varphi} \mid \varphi(x, e)=\{x\}, x \neq e\right\} \\
D_{\varphi} & :=\left\{x \in I_{\varphi} \mid \varphi(x, e)=\{x, e\}\right\} \\
R_{\varphi} & :=\left\{x \in I_{\varphi} \mid \varphi(x, e)=\{e\}, x \neq e\right\} .
\end{aligned}
$$

Now let $I$ be an arbitrary Abelian multiplicative group. An LDR decomposition of $I$ is a partition of $I$ into sets $L, D$ and $R$ such that $L, D$ and $R$ are closed under multiplication, $D$ is not empty, $\lambda \in R$ and $\mu \in D$ imply that $\lambda \mu \in R$, and $\lambda \in L$ and $\mu \in D$ imply that $\lambda \mu \in L$. Automatically we have $e \in D$ (where $e$ denotes the neutral element of $I$ ), and if $x \in L$ then $\frac{e}{x} \in R$ (where $\frac{e}{x}$ denotes the inverse of $x$ ).

Theorem 4.1 The sets $L_{\varphi}, D_{\varphi}$ and $R_{\varphi}$ form an LDR decomposition of the Abelian multiplicative group $I_{\varphi}$.

Proof. Clearly $L_{\varphi}, D_{\varphi}$ and $R_{\varphi}$ partition $I_{\varphi}$. Further, since $\varphi$ is transitive and satisfies COV, $L_{\varphi}, D_{\varphi}$ and $R_{\varphi}$ are closed under multiplication. $D_{\varphi}$ is not empty because it contains $e$.

Now take an $x \in R_{\varphi}$ and $y \in D_{\varphi}$. So, using COV, $e \in \varphi(x, e)$ and $x \in \varphi(x y, x)$. Hence, by transitivity, $e \in \varphi(x y, e)$. However, also $x y \notin \varphi(x y, y)$ and $e \in \varphi(y, e)$. Hence, by transitivity, $x y \notin \varphi(x y, e)$. Thus, $\varphi(x y, e)=\{e\}$ and $x y \in R_{\varphi}$.

Similarly it can be shown that $x \in L_{\varphi}$ and $y \in D_{\varphi}$ imply $x y \in L_{\varphi}$. Hence, $L_{\varphi}, D_{\varphi}$ and $R_{\varphi}$ form an LDR decomposition of $I_{\varphi}$.

Thus, each WPO, IIA and COV bargaining solution $\varphi$ determines a quadruple

$$
\left(\alpha, L_{\varphi}, D_{\varphi}, R_{\varphi}\right)
$$


where $\alpha \geq 0$ is such that the set

$$
I_{\alpha}:=\left\{x \in \mathbb{R}_{++}^{n} \mid \prod_{i} x_{i}^{\alpha_{i}}=1\right\}
$$

equals $I_{\varphi}$, and $L_{\varphi}, D_{\varphi}$ and $R_{\varphi}$ constitute an LDR decomposition of $I_{\alpha}$. We show in this section that this quadruple characterizes the solution $\varphi$ in the following sense. Not only does each WPO, IIA and COV bargaining solution determine a unique quadruple, but conversely also each such a quadruple uniquely determines a WPO, IIA and COV bargaining solution.

In order to prove this, let $\alpha=\left(\alpha_{1}, \ldots, \alpha_{n}\right)$ be a non-negative vector with $\sum_{i} \alpha_{i}=1$. It is easy to check that $I_{\alpha}$ is an Abelian multiplicative group. Let $L_{\alpha}, D_{\alpha}$ and $R_{\alpha}$ form an LDR decomposition of $I_{\alpha}$.

Theorem 4.2 There exists a unique WPO, COV and IIA solution $\varphi: \mathcal{F} \rightarrow \mathbb{R}_{++}^{n}$ such that $I_{\varphi}=I_{\alpha}, L_{\varphi}=L_{\alpha}, D_{\varphi}=D_{\alpha}$ and $R_{\varphi}=R_{\alpha}$.

Proof. First take an arbitrary set $\{x, y\} \in \mathcal{F}$. Take $\lambda>0$ such that $\lambda \frac{x}{y} \in I_{\alpha}$. Define $\mu(x, y)$ by

$$
\mu(x, y):= \begin{cases}\{x\} & \text { if } \lambda<1 \\ \{x\} & \text { if } \lambda=1 \text { and } \frac{x}{y} \in L_{\alpha} \\ \{x, y\} & \text { if } \lambda=1 \text { and } \frac{x}{y} \in D_{\alpha} \\ \{y\} & \text { if } \lambda=1 \text { and } \frac{x}{y} \in R_{\alpha} \\ \{y\} & \text { if } \lambda>1 .\end{cases}
$$

Observe that $\mu(x, y)$ is defined in accordance with what the LDR decomposition $L_{\alpha}, D_{\alpha}, R_{\alpha}$ of $I_{\alpha}$ prescribes for the set $\left\{\frac{x}{y}, e\right\}$. By COV, this also implies how to make the choice for $\{x, y\}$.

We argue that $\mu$ is transitive. Take $x^{1}, x^{2}, x^{3} \in \mathbb{R}_{++}^{n}$. Suppose that $x^{2} \in \mu\left(x^{1}, x^{2}\right)$ and $x^{3} \in \mu\left(x^{2}, x^{3}\right)$. Let $\lambda_{1}>0$ and $\lambda_{2}>0$ be such that $\lambda_{1} \frac{x^{1}}{x^{2}} \in I_{\alpha}$ and $\lambda_{2} \frac{x^{2}}{x^{3}} \in I_{\alpha}$. Then, since $I_{\alpha}$ is a multiplicative group, $\lambda_{1} \lambda_{2} \frac{x^{1}}{x^{3}} \in I_{\alpha}$. Moreover, since $x^{2} \in \mu\left(x^{1}, x^{2}\right)$ and $x^{3} \in \mu\left(x^{2}, x^{3}\right)$, we have that $\lambda_{1} \geq 1$ and $\lambda_{2} \geq 1$. Therefore also $\lambda_{1} \lambda_{2} \geq 1$. We distinguish two cases.

(a) $\lambda_{1} \lambda_{2}>1$. In this case $\mu\left(x^{1}, x^{3}\right)=\left\{x^{3}\right\}$ by definition.

(b) $\lambda_{1} \lambda_{2}=1$. In this case $\lambda_{1}=\lambda_{2}=1$. So, by definition of $\mu, \frac{x^{1}}{x^{2}} \in R_{\alpha} \cup D_{\alpha}$ and $\frac{x^{2}}{x^{3}} \in R_{\alpha} \cup D_{\alpha}$. Then, because $L_{\alpha}, D_{\alpha}, R_{\alpha}$ is an LDR decomposition of $I_{\alpha}$, also $\frac{x^{1}}{x^{3}} \in R_{\alpha} \cup D_{\alpha}$, and hence $x^{3} \in \mu\left(x^{1}, x^{3}\right)$.

Next define $\varphi: \mathcal{F} \rightarrow \mathbb{R}_{++}^{n}$ by, for all $F \in \mathcal{F}$

$$
\varphi(F):=\{x \in F \mid x \in \mu(x, y) \text { for all } y \in F\}
$$

We show that $\varphi$ is the unique WPO, IIA and COV bargaining solution $\varphi: \mathcal{F} \rightarrow \mathbb{R}_{++}^{n}$ such that $\varphi(x, y)=\mu(x, y)$ for all $x, y \in \mathbb{R}_{++}^{n}$. 
A. From the definition of $\varphi$ it follows immediately that $\varphi(x, y)=\mu(x, y)$ for all $x, y \in \mathbb{R}_{++}^{n}$. From this it follows that $I_{\varphi}=I_{\alpha}, L_{\varphi}=L_{\alpha}, D_{\varphi}=D_{\alpha}$ and $R_{\varphi}=R_{\alpha}$ (once we established that $\varphi$ is a bargaining solution and that it satisfies WPO, IIA, and COV), since all these sets are defined by considering pairs of elements in $\mathbb{R}_{++}^{n}$.

B. Take $F \in \mathcal{F}$. We claim that $\varphi(F)$ is not empty. Suppose it were empty. Take a point $x_{1} \in F$. Since $x_{1} \notin \varphi(F)$ there is an $x_{2} \in F$ with $x_{1} \notin \mu\left(x_{1}, x_{2}\right)$. Since $\mu\left(x_{1}, x_{2}\right)$ is not empty, necessarily $\left\{x_{2}\right\}=\mu\left(x_{1}, x_{2}\right)$. In the same way we find an $x_{3} \in F$ with $x_{2} \notin \mu\left(x_{2}, x_{3}\right)$. Iterating this procedure yields a sequence $x_{1}, x_{2}, x_{3}, \ldots$ in $F$ with $x_{k} \notin \mu\left(x_{k}, x_{k+1}\right)$ for all $k \geq 1$. Since $F$ is finite, we have $x_{r}=x_{t}$ for some $r, t$ with $r<t$. This violates the transitivity of $\mu$.

C. The solution $\varphi$ satisfies IIA. Take $F, G \in \mathcal{F}$ with $G \subset F$ and $G \cap \varphi(F) \neq \phi$. We show that $\varphi(G)=G \cap \varphi(F)$.

C1. Take an $x \in \varphi(G)$. Clearly $x \in G$. In order to show that $x \in \varphi(F)$, take $y \in F$. If $x \in \mu(x, y)$ we are done. Since $G \cap \varphi(F) \neq \phi$, we can take $z \in G \cap \varphi(F)$. Since $x \in \mu(x, z)$ and $z \in \mu(y, z)$, we have $x \in \mu(x, y)$ by the transitivity of $\mu$.

C2. Take an $x \in G \cap \varphi(F)$. Take a $y \in G$. Then $x \in \mu(x, y)$ since $x \in \varphi(F)$. Hence $x \in \varphi(G)$.

D. Next we show that $\varphi$ satisfies WPO. Take $F \in \mathcal{F}$ and $x, y \in F$ with $x<y$. Then $\frac{x}{y}<e$. So, for $\lambda>0$ with $\lambda \frac{x}{y} \in I_{\alpha}$ we have $\lambda>1$. Hence, $\mu(x, y)=\{y\}$ and therefore $x \notin \varphi(F)$ by the definition of $\varphi$.

E. We show that $\varphi$ satisfies COV. Take $a \in \mathbb{R}_{++}^{n}$, take $F \in \mathcal{F}$ and take $x \in \varphi(F)$. It suffices to show that $a x \in \varphi(a F)$. Take an arbitrary $y \in a F$. Then $\frac{y}{a} \in F$. So, $x \in \mu\left(x, \frac{y}{a}\right)$. Hence, $a x \in \mu(a x, y)$ by the definition of $\mu$.

F. Finally we prove uniqueness. Let $\psi$ be an arbitrary WPO, IIA and COV bargaining solution such that $\psi(x, y)=\mu(x, y)$ for all $x, y \in \mathbb{R}_{++}^{n}$. Take $F \in \mathcal{F}$. We prove that $\psi(F)=$ $\{x \in F \mid x \in \mu(x, y)$ for all $y \in F\}$.

F1. Take an $x \in \psi(F)$. Take a $y \in F$. Then, from IIA and the fact that $\psi(x, y)=\mu(x, y)$, it follows that $x \in \mu(x, y)$.

F2. Take an $x \in F$ with $x \in \mu(x, y)$ for all $y \in F$. Since $\psi(F)$ is not empty, we can take $z \in \psi(F)$. Then $x \in \mu(x, z)=\psi(x, z)$, and hence $x \in \psi(F)$ by IIA. 


\section{Applications}

In this section we discuss a few implications of our characterization. First we show that the class of Nash bargaining solutions is characterized by WPO, IIA, COV and CON, and that the symmetric Nash bargaining solution results if CON is replaced by SYM. Then we show how our characterization can be used to construct new bargaining solutions that satisfy WPO, IIA and COV.

\subsection{An axiomatization of Nash bargaining solutions}

As a direct application of our results we can now easily prove that the class of Nash bargaining solutions is fully characterized by WPO, IIA, COV and CON. Notice that the $\alpha$-Nash bargaining solution corresponds to the choice $D_{\alpha}=I_{\alpha}$ and $L_{\alpha}=R_{\alpha}=\phi$. Using this observation it is easy to prove the following theorem.

Theorem 5.1 A solution $\varphi$ on $\mathcal{F}$ is a Nash bargaining solution if and only if $\varphi$ satisfies WPO, IIA, COV and $\mathrm{CON}$.

Proof. Let $\varphi$ be a bargaining solution that satisfies WPO, IIA, COV and CON. We show that $D_{\varphi}=I_{\varphi}$. Take an $x \in I_{\varphi}$. Then, for any positive $\lambda<1, \varphi(\lambda x, e)=\{e\}$ by Lemma 3.1. Hence, by CON, $e \in \varphi(x, e)$. Conversely, for any $\lambda>1, \varphi(\lambda x, e)=\{\lambda x\}$ by Lemma 3.1. Hence, by CON, $x \in \varphi(x, e)$.

Imposing SYM instead of CON singles out the symmetric Nash bargaining solution $N^{\alpha}$ with $\alpha_{i}=1 / n$ for every $i$.

Theorem 5.2 A solution $\varphi$ on $\mathcal{F}$ is the symmetric Nash bargaining solution if and only if $\varphi$ satisfies WPO, IIA, COV and SYM.

Proof. We only prove the if-direction. Let $\varphi$ be a bargaining solution with these properties. Obviously, $\alpha_{i}=1 / n$ for all $i$. Take $x \in I_{\varphi}$. It is sufficient to prove that $x \in D_{\varphi}$. It is not difficult (cf. Xu and Yoshihara [11]) to find an $a \in \mathbb{R}_{++}^{n}$ such that $a=a e$ can be obtained from $a x$ by a permutation of coordinates. Consider the finite bargaining problem $B$ consisting of $a x$ and all vectors obtainable from $a x$ by a permutation of coordinates. By $\operatorname{SYM}, \varphi(B)=B$. In particular, $a, a x \in \varphi(B)$. By COV and IIA, $\varphi(x, e)=\{x, e\}$, hence $x \in D_{\varphi}$.

\subsection{Discontinuous bargaining solutions}

In this subsection we use the characterization in Theorems 4.1 and 4.2 to construct discontinuous WPO, IIA and COV bargaining solutions. Take a non-negative vector $\alpha \in \mathbb{R}^{n}$ with 
$\sum_{i} \alpha_{i}=1$. Since the choice $D_{\alpha}=I_{\alpha}$ and $L_{\alpha}=R_{\alpha}=\phi$ corresponds to the $\alpha$-Nash solution $N^{\alpha}$, by Theorem 5.1 any other choice of $D_{\alpha}$ necessarily induces a bargaining solution that is discontinuous. We discuss two techniques to construct such discontinuous WPO, IIA and COV bargaining solutions.

TeChnique I We construct the sets $L_{\alpha}, D_{\alpha}$ and $R_{\alpha}$ as follows. For $1 \leq k \leq n$, write

$$
G_{k}:=\left\{x \in I_{\alpha} \mid x_{i}=1 \text { for all } i<k, \text { and } x_{k}>1\right\}
$$

and

$$
S_{k}:=\left\{x \in I_{\alpha} \mid x_{i}=1 \text { for all } i<k \text {, and } x_{k}<1\right\} .
$$

Notice that $I_{\alpha}$ is the disjoint union of $\{e\}$ and the sets $G_{k}$ and $S_{k}$. Define, for $l \leq n$,

$$
L_{\alpha}:=\bigcup_{k=1}^{l} L_{k} \quad \text { and } \quad R_{\alpha}:=\bigcup_{k=1}^{l} R_{k} \quad \text { and } \quad D_{\alpha}:=\{e\} \cup \bigcup_{k=l+1}^{n}\left(G_{k} \cup S_{k}\right)
$$

where for each $k \leq l$ the sets $L_{k}$ and $R_{k}$ are chosen from $\left\{G_{k}, S_{k}\right\}$ in such a way that $\left\{L_{k}, R_{k}\right\}=$ $\left\{G_{k}, S_{k}\right\}$. In other words, $L_{\alpha}$ is constructed by choosing for each $k \leq l$ exactly one of the sets $G_{k}$ and $S_{k}$ and taking the union of these choices. $R_{\alpha}$ consists of the union of the complementary sets, while $D_{\alpha}$ consists of the remaining sets for $k>l$, together with the vector $e$.

As an extreme example of the class of bargaining solutions thus constructed, notice that the choice $D_{\alpha}=\{e\}$ corresponds to the class of single-valued WPO, IIA and COV bargaining solutions, since all ties within $I_{\alpha}$ get broken, either in favor of $x \in I_{\alpha}$ (when $x \in L_{\alpha}$ ) or in favor of $e$ (when $x \in R_{\alpha}$ ). In other words, in this case for any $x \in I_{\alpha}$, either $\varphi(x, e)=\{x\}$ or $\varphi(x, e)=\{e\}$. If for example $L_{k}=G_{k}$ for all $k$, then the bargaining solution induced by $\alpha$, $D_{\alpha}=\{e\}$ and $L_{\alpha}$ and $R_{\alpha}$ is the single-valued solution that, for a bargaining problem $F \in \mathcal{F}$, results from first maximizing the Nash product $\prod_{i} x_{i}^{\alpha_{i}}$ over $F$, and within the remaining set consecutively maximizing $x_{1}, x_{2}, \ldots, x_{n}$ until only one point remains.

More generally, the solutions constructed here are in fact those solutions that result by first maximizing a Nash product over the initial bargaining problem $F$, and then consecutively maximizing or minimizing $l$ coordinates. We could generalize the construction presented here even further to include iterated maximization of arbitrary Nash products. For ease of exposition we restricted ourselves to consecutive maximization (or minimization) of single coordinates (a special type of Nash product that corresponds to the choice where $\alpha$ is a unit vector).

TECHNIQUE II The second technique to construct WPO, IIA and COV bargaining solutions 
is more general. First, let $H_{\alpha} \subset \mathbb{R}^{n}$ be defined by

$$
H_{\alpha}:=\left\{x \in \mathbb{R}^{n} \mid \sum_{i} \alpha_{i} x_{i}=0\right\}
$$

Any additive functional $f: H_{\alpha} \rightarrow \mathbb{R}$ defines an LDR decomposition as follows. Note that the operation $\rho: I_{\alpha} \rightarrow H_{\alpha}$ defined by

$$
\rho\left(x_{1}, \ldots, x_{n}\right):=\left(\ln \left(x_{1}\right), \ldots, \ln \left(x_{n}\right)\right)
$$

is an isomorphism w.r.t. addition on $H_{\alpha}$ and multiplication on $I_{\alpha}$. Define

$$
\begin{aligned}
L_{f} & :=\left\{x \in I_{\alpha} \mid(f \circ \rho)(x)<0\right\} \\
D_{f} & :=\left\{x \in I_{\alpha} \mid(f \circ \rho)(x)=0\right\} \\
R_{f} & :=\left\{x \in I_{\alpha} \mid(f \circ \rho)(x)>0\right\} .
\end{aligned}
$$

It is easy to check that $L_{f}, D_{f}$ and $R_{f}$ form an LDR decomposition of $I_{\alpha}$. Thus, every additive functional $f$ induces a WPO, IIA and COV bargaining solution $\varphi(f)$, and two such functionals $f$ and $g$ induce different solutions $\varphi(f)$ and $\varphi(g)$ as soon as $L_{f}, D_{f}, R_{f}$ is a different LDR decomposition than $L_{g}, D_{g}, R_{g}$. Also notice that $D_{f}=I_{\alpha}$ precisely when $f \equiv 0$ on $H_{\alpha}$. Consequently, any non-zero additive functional induces a discontinuous WPO, IIA and COV bargaining solution.

Now we use the above observation to construct a plethora of WPO, IIA and COV bargaining solutions as follows. Of course every linear functional $f: H_{\alpha} \rightarrow \mathbb{R}$ induces a solution $\varphi(f)$. However, using Zorn's Lemma, we can construct many, many more different additive functionals, and hence many, many more different WPO, IIA and COV bargaining solutions. First we need some notation. Let $X$ be a non-empty set and let $\preceq$ be a binary relation on $X$ that satisfies

[1] (reflexivity) for all $x \in X$ we have $x \preceq x$, and

[2] (transitivity) for all $x, y, z \in X$ we have $x \preceq z$ whenever both $x \preceq y$ and $y \preceq z$.

We say that $\preceq$ is a partial order on $X$. A subset $C$ of $X$ is called a chain if for any two elements $x$ and $y$ of $C$ we have at least one of the two inequalities $x \preceq y$ and $y \preceq x$. A chain $C$ of $X$ is said to have an upper bound if there exists an element $a$ of $X$ such that $x \preceq a$ for all $x$ in $C$. An element $a$ of $X$ is called maximal if for any $x$ in $X$ the inequality $a \preceq x$ only holds if $x \preceq a$ holds as well. 
Lemma 5.3 (Zorn) Suppose that every chain of $X$ has an upper bound. Then $X$ has a maximal element.

Using Zorn's lemma we can easily prove the following result, due to Hamel.

Theorem $5.4 \mathbb{R}^{n}$ has a basis over $\mathbb{Q}$. Consequently, also $H_{\alpha}$ has a basis over $\mathbb{Q}$.

Proof. $\quad$ Let $X$ be the set of $\mathbb{Q}$-independent sets in $\mathbb{R}^{n}$, ordered by set inclusion. Let $C$ be a chain in $X$. Then $\cup_{B \in C} B$ is an upper bound of $C$. Hence, by Zorn's Lemma, $X$ has a maximal element, say $B$. It is straightforward to prove that $B$ is a basis of $\mathbb{R}^{n}$ over $\mathbb{Q}$. The second part of the theorem follows from the observation that $H_{\alpha}$ is linearly isomorphic to $\mathbb{R}^{n-1}$.

We construct a multitude of WPO, IIA and COV bargaining solutions as follows. Let $B$ be a basis of $H_{\alpha}$ over $\mathbb{Q}$. An assignment for $B$ is a function $f: B \rightarrow \mathbb{R}$. It is clear that every assignment $f: B \rightarrow \mathbb{R}$ uniquely extends to an additive functional $f: H_{\alpha} \rightarrow \mathbb{R}$. ${ }^{5}$ Thus, there are as many additive functionals on $H_{\alpha}$ as there are assignments on $B$. We already observed that each additive functional induces a WPO, IIA and COV bargaining solution. Hence, we have the following theorem.

Theorem 5.5 For every assignment $f$ for $B$ there exists a unique WPO, IIA and COV bargaining solution $\varphi(f)$ such that $L_{f}=L_{\varphi(f)}, D_{f}=D_{\varphi(f)}$ and $R_{f}=R_{\varphi(f)}$. Consequently, for any two assignments $f$ and $g, \varphi(f) \neq \varphi(g)$ precisely when $\left(L_{f}, D_{f}, R_{f}\right) \neq\left(L_{g}, D_{g}, R_{g}\right)$.

Note that $\varphi(f)$ might still be equal to $\varphi(g)$, even when $f \neq g$ (for example, when $g=2 f$ ). Nevertheless, the qualification 'plethora' still applies for the following reason. Notice that $B$ is uncountable. Further, $\left(L_{f}, D_{f}, R_{f}\right)$ is a different LDR decomposition of $I_{\alpha}$ than $\left(L_{g}, D_{g}, R_{g}\right)$ at least when there is a $b \in B$ for which for example $f(b)>0$ and $g(b) \leq 0$ (because in that case $\rho^{-1}(b) \in R_{f}$ while $\left.\rho^{-1}(b) \notin R_{g}\right)$. Thus the number of different LDR decompositions that can be constructed this way is still at least as large as the cardinality of $3^{B}$.

\section{Two player bargaining problems}

The characterization of WPO, IIA and COV bargaining solutions works particularly elegant in the case of two player bargaining problems. For any vector $(\alpha, 1-\alpha)$ with $0 \leq \alpha \leq 1$, either $D_{\alpha}=I_{\alpha}$, or $D_{\alpha}=\{e\}$, or $D_{\alpha}$ is a strict subset of $I_{\alpha}$. In the latter case, however, it is easy to show, using COV and transitivity, that $D_{\alpha}$ is dense in $I_{\alpha}$.

\footnotetext{
${ }^{5}$ Formally we should use a different symbol to indicate the additive function, but we chose to use a slight abuse of notation here.
} 
The option $D_{\alpha}=I_{\alpha}$ corresponds again to the $\alpha$-Nash bargaining solution. For the option $D_{\alpha}=\{e\}$, we have at least the following two possibilities.

In the first possibility we let $L_{\alpha}=\left\{\begin{array}{ll}\left\{\left(x_{1}, x_{2}\right) \in I_{\alpha} \mid x_{2}<1\right\} & \text { if } \alpha>0 \\ \left\{\left(x_{1}, x_{2}\right) \in I_{\alpha} \mid x_{1}>1\right\} & \text { if } \alpha=0\end{array} \quad\right.$ and $\quad R_{\alpha}=\left\{\begin{array}{l}\left\{\left(x_{1}, x_{2}\right) \in I_{\alpha} \mid x_{2}>1\right\} \quad \text { if } \alpha>0 \\ \left\{\left(x_{1}, x_{2}\right) \in I_{\alpha} \mid x_{1}<1\right\} \quad \text { if } \alpha=0\end{array}\right.$ This choice corresponds to the single-valued bargaining solution, denoted $N^{\alpha, R}$, which for $\alpha>0$ and a bargaining problem $F \subset \mathbb{R}_{++}^{2}$, results from first maximizing the function $x_{1}^{\alpha} x_{2}^{1-\alpha}$ over $F$, and subsequently minimizing the second coordinate over the remaining alternatives. For $\alpha=0$, the solution results from first maximizing the second coordinate and, subsequently, maximizing the first coordinate.

The second possibility is to interchange the definitions of $L_{\alpha}$ and $R_{\alpha}$ in the above display. This choice corresponds to the single-valued bargaining solution, denoted $N^{\alpha, L}$, which for $\alpha<1$ and a bargaining problem $F \subset \mathbb{R}_{++}^{2}$, results from first maximizing the function $x_{1}^{\alpha} x_{2}^{1-\alpha}$ over $F$, and subsequently minimizing the first coordinate over the remaining alternatives. For $\alpha=1$, the solution results from first maximizing the first coordinate and, subsequently, maximizing the second coordinate.

As discussed in the previous section, there are many more possible solutions once we use the Axiom of Choice (AoC). The above-mentioned bargaining solutions, however, are to our knowledge all solutions one can construct using classical tools, i.e., the Zermelo-Fraenkel axioms of set theory $(\mathrm{ZF})$. In particular, we conjecture that solutions $\varphi$ with $\{e\} \subsetneq D_{\varphi} \subsetneq I_{\varphi}$ cannot be constructed without AoC. We discuss the reasons for this conjecture in somewhat more detail. Analogous to the notion of an LDR decomposition in the multiplicative case, a PZN decomposition of $\mathbb{R}$ is a partition of $\mathbb{R}$ into sets $P, Z$ and $N$ such that $P, Z$ and $N$ are closed under addition, $Z$ is not empty, $x \in N$ and $y \in Z$ implies that $x+y \in N$, and $x \in P$ and $y \in Z$ implies that $x+y \in P$.

Theorem 6.1 There exists a WPO, IIA and COV bargaining solution $\varphi: \mathcal{F} \rightarrow \mathbb{R}_{++}^{2}$ with $\{e\} \subsetneq D_{\varphi} \subsetneq I_{\alpha}$ if and only if there exists a PZN decomposition $P, Z$ and $N$ of $\mathbb{R}$ with $\{0\} \subsetneq Z \subsetneq \mathbb{R}$.

Proof. If $\alpha<1$, the map $\pi: I_{\alpha} \rightarrow \mathbb{R}$ defined by

$$
\pi\left(x_{1}, x_{1}^{\frac{-\alpha}{1-\alpha}}\right):=\log x_{1}
$$

is an isomorphism that maps LDR decompositions of $I_{\alpha}$ to PZN decompositions of $\mathbb{R}$ and vice versa. In case $\alpha=1$, we can take $\pi\left(1, x_{2}\right):=\log x_{2}$ to be the isomorphism. 
The existence of a PZN decomposition of $\mathbb{R}$ is conjectured to be independent of the ZF axioms without AoC. It is for example implied by, but perhaps weaker than, the existence of a discontinuous additive function on $\mathbb{R}$, which in turn is implied by, but perhaps weaker than, the existence of a basis for $\mathbb{R}$ over $\mathbb{Q}$ as in Theorem 5.4. To our knowledge, Theorem 5.4 has thus far only been proved using AoC, and it has not been shown to be an independent statement ${ }^{6}$. Thus, in connection with the case where $\{e\} \subsetneq D_{\alpha} \subsetneq I_{\alpha}$, for two-person bargaining problems we conjecture that the statement

There exists a non-single-valued and discontinuous WPO, COV and IIA bargaining solution is independent of the ZF axioms of set theory without AoC.

\section{Larger domains}

So far we have only considered the domain of all $n$-person finite bargaining problems. In this section we show that the characterization of the class of Nash bargaining solutions (Theorem 5.1) by WPO, COV, IIA and CON extends directly to larger domains of compact bargaining problems (Theorem 7.1). Replacing CON by SYM results in a characterization of the symmetric Nash bargaining solution (Theorem 7.2). We also show that for certain domains, in particular the domain of all compact bargaining problems, the characterization of all WPO, COV and IIA bargaining solutions does not entirely go through (Theorem 7.3). This has particular consequences for the two-person case (Theorem 7.4).

\subsection{Nash bargaining solutions}

We show that the class of Nash bargaining solutions is characterized by WPO, COV, IIA and CON on any domain $\mathcal{D}$ containing the domain of finite bargaining problems. ${ }^{7}$

Theorem 7.1 Let $\mathcal{F} \subset \mathcal{D} \subset \mathcal{C}$ and let $\varphi: \mathcal{D} \rightarrow \mathbb{R}_{++}^{n}$ be a bargaining solution. Then $\varphi$ satisfies WPO, COV, IIA and CON if and only if $\varphi$ is a Nash bargaining solution.

Proof. We only prove the only-if direction. Let $\varphi$ satisfy the axioms in the theorem. Let $\varphi_{\mathcal{F}}: \mathcal{F} \rightarrow \mathbb{R}_{++}^{n}$ denote the restriction of $\varphi$ to $\mathcal{F}$. Obviously $\varphi_{\mathcal{F}}$ also satisfies WPO, COV, IIA and CON. Thus, by Theorem 5.1 there exists a non-zero vector $\alpha \in \mathbb{R}_{+}^{n}$ such that $\varphi(F)=$ $N^{\alpha}(F)$ for all $F \in \mathcal{F}$. Take a $B \in \mathcal{D}$. We show that $\varphi(B)=N^{\alpha}(B)$.

\footnotetext{
${ }^{6}$ It is known is that the statement 'Every vector space has a basis' is equivalent to AoC, and hence an independent statement. This does not exclude that bases for specific vector spaces, such as $\mathbb{R}^{n}$ over $\mathbb{R}$, can be constructed without using AoC.

${ }^{7}$ With some slight modifications this assumption can easily be relaxed, e.g., by assuming that $\mathcal{D}$ contains all finitely generated comprehensive problems.
} 
Take an $x \in \varphi(B)$. Suppose that $x \notin N^{\alpha}(B)$. Then there is a $y \in B$ with $\prod_{i} x_{i}^{\alpha_{i}}<\prod_{i} y_{i}^{\alpha_{i}}$. So, $\varphi(x, y)=\varphi_{\mathcal{F}}(x, y)=\{y\}$, which contradicts IIA. Hence, $x \in N^{\alpha}(B)$.

Conversely, suppose that $x \in N^{\alpha}(B)$. Since $\varphi(B)$ is not empty, we can take $y \in \varphi(B)$. Then also $y \in N^{\alpha}(B)$ by the previous argument. So $\prod_{i} x_{i}^{\alpha_{i}}=\prod_{i} y_{i}^{\alpha_{i}}$, and then $\varphi(x, y)=\varphi_{\mathcal{F}}(x, y)=$ $\{x, y\}$. Hence, $x \in \varphi(B)$ by IIA.

By an analogous proof based on Theorem 5.2, we obtain a characterization of the symmetric Nash bargaining solution.

Theorem 7.2 Let $\mathcal{F} \subset \mathcal{D} \subset \mathcal{C}$ and let $\varphi: \mathcal{D} \rightarrow \mathbb{R}_{++}^{n}$ be a bargaining solution. Then $\varphi$ satisfies WPO, COV, IIA and SYM if and only if $\varphi$ is the symmetric Nash bargaining solution.

\subsection{WPO, COV and IIA bargaining solutions}

More surprisingly, the characterization of all WPO, COV and IIA bargaining solutions in terms of a non-zero vector $\alpha \in \mathbb{R}_{++}^{n}$ and an LDR decomposition of $I_{\varphi}$ does not extend straightforwardly. It is still true that each WPO, COV and IIA bargaining solution on a domain $\mathcal{D}$ induces a non-zero vector $\alpha \in \mathbb{R}_{++}^{n}$ and an LDR decomposition of $I_{\varphi}$. The converse, however, no longer holds in general.

We study the question which WPO, COV and IIA bargaining solutions on $\mathcal{F}$ can be extended to the domain $\mathcal{C}$ of all compact bargaining problems. We take $\mathcal{C}$ for convenience of presentation: it is not hard to check that the results below also hold for specific smaller domains. Moreover, $\mathcal{C}$ is the domain of not necessarily convex bargaining problems that is usually considered.

In order to answer this question we need the following observation. Take a non-zero vector $\alpha \in \mathbb{R}_{+}^{n}$ and let $L_{\alpha}, D_{\alpha}$ and $R_{\alpha}$ be an LDR decomposition of $I_{\alpha}$.

Theorem 7.3 Suppose that $L_{\alpha}$ is dense in $I_{\alpha}$. Then there does not exist a WPO, COV and IIA bargaining solution $\varphi: \mathcal{C} \rightarrow \mathbb{R}_{++}^{n}$ such that $I_{\varphi}=I_{\alpha}, L_{\varphi}=L_{\alpha}, D_{\varphi}=D_{\alpha}$ and $R_{\varphi}=R_{\alpha}$.

Proof. For $x, y \in \mathbb{R}^{n}$, let $d(x, y)$ denote the Euclidean distance between $x$ and $y$. For ease of exposition we use the isomorphism $\rho: I_{\alpha} \rightarrow H_{\alpha}$ defined in subsection 5.2. Since $L_{\alpha}$ is dense in $I_{\alpha}$, it follows in particular that $R_{\alpha}$ is not empty. Take an $r \in R_{\alpha}$. Since $L_{\alpha}$ is dense in $I_{\alpha}$ we can take an $l_{1} \in L_{\alpha}$ with $d\left(\rho(r), \rho\left(l_{1}\right)\right)<\frac{1}{2}$. Iteratively suppose for $m \in \mathbb{N}, m \geq 2$ that $l_{1}, \ldots, l_{m-1}$ have been chosen. Since $r \prod_{k=1}^{m-1} l_{k}^{-1}$ is an element of $I_{\alpha}$, we can take an $l_{m} \in L_{\alpha}$ such that

$$
d\left(\rho\left(r \prod_{k=1}^{m-1} l_{k}^{-1}\right), \rho\left(l_{m}\right)\right)<\left(\frac{1}{2}\right)^{m}
$$


Write $L_{m}:=\prod_{k=1}^{m} l_{m}$ for each $m \in \mathbb{N}$. Then, by translation invariance and preservation of group structure by $\rho$,

$$
\begin{aligned}
d\left(\rho(r), \rho\left(L_{m}\right)\right) & =d\left(\rho(r), \sum_{k=1}^{m} \rho\left(l_{k}\right)\right) \\
& =d\left(\rho(r)-\sum_{k=1}^{m-1} \rho\left(l_{k}\right), \rho\left(l_{m}\right)\right) \\
& =d\left(\rho\left(r \prod_{k=1}^{m-1} l_{k}^{-1}\right), \rho\left(l_{m}\right)\right)<\left(\frac{1}{2}\right)^{m}
\end{aligned}
$$

In particular

$$
B:=\left\{e, r, L_{1}, L_{2}, \ldots\right\}
$$

is a compact set. Thus, $B \in \mathcal{C}$ and $B \subset I_{\alpha}$.

Now let $\varphi: \mathcal{C} \rightarrow \mathbb{R}_{++}^{n}$ be a WPO, COV and IIA bargaining solution. We argue that the assumptions $I_{\varphi}=I_{\alpha}, L_{\varphi}=L_{\alpha}, D_{\varphi}=D_{\alpha}$ and $R_{\varphi}=R_{\alpha}$ contradict the non-emptiness of $\varphi(B)$. Firstly notice that $\varphi\left(e, L_{1}\right)=\left\{L_{1}\right\}$ because $L_{1}=l_{1}$ is an element of $L_{\alpha}=L_{\varphi}$. Hence, $e \notin \varphi(B)$ by IIA. Secondly notice that $\varphi(e, r)=\{e\}$ because $r$ is an element of $R_{\alpha}=R_{\varphi}$. Hence, $r \notin \varphi(B)$ by IIA. Thirdly, for any $m \in \mathbb{N}$, by $\mathrm{COV}$ we have

$$
\varphi\left(L_{m}, L_{m+1}\right)=L_{m} \cdot \varphi\left(e, l_{m+1}\right)=L_{m} \cdot\left\{l_{m+1}\right\}=\left\{L_{m+1}\right\}
$$

because $l_{m+1}$ is an element of $L_{\alpha}=L_{\varphi}$. Hence, for any $m \in \mathbb{N}, L_{m} \notin \varphi(B)$ by IIA.

Thus, none of the elements of $B$ can be an element of $\varphi(B)$, which contradicts the non-emptiness of $\varphi(B)$.

For two-person bargaining problems Theorem 7.3 results in a complete description of all WPO, COV and IIA bargaining solutions.

Theorem 7.4 Let $\varphi: \mathcal{C} \rightarrow \mathbb{R}_{++}^{2}$ be a WPO, COV and IIA two-person bargaining solution. Then there exists a non-zero vector $\alpha \in \mathbb{R}_{+}^{2}$ such that $\varphi$ either equals $N^{\alpha}$, or $\varphi$ is single-valued and $\varphi$ either equals $N^{\alpha, L}$ or $N^{\alpha, R}$.

Proof. By Corollary 3.4 there exists a non-zero vector $\alpha \in \mathbb{R}_{+}^{2}$ such that $\varphi$ is a refinement of $N^{\alpha}$.

Suppose $\varphi \neq N^{\alpha}$. Then $D_{\varphi} \neq I_{\varphi}$. First we show that $\varphi$ is single-valued. Suppose, to the contrary, that also $\{e\} \neq D_{\varphi}$. Since $n=2$, by COV and transitivity this is easily seen to imply 
that $D_{\varphi}$ is dense in $I_{\varphi}$. Since $L_{\varphi} \neq \phi$, we can take $l \in L_{\varphi}$. Then $l D_{\varphi}$ is a subset of $L_{\varphi}$ that is dense in $I_{\varphi}$. Hence, $L_{\varphi}$ itself is dense in $I_{\varphi}$. This contradicts Theorem 7.3.

So, $\varphi$ is single-valued, and $D_{\varphi}=\{e\}$. Take an $\left(x_{1}, x_{2}\right) \in L_{\varphi}$. Suppose $\alpha_{1}>$ 0. W.l.o.g. $x_{2}<1$. We show that $L_{\varphi}=\left\{\left(x_{1}, x_{2}\right) \in I_{\alpha} \mid x_{2}<1\right\}$. If this is not the case, then there is a $\left(y_{1}, y_{2}\right) \in L_{\varphi}$ with $y_{2}>1$. But then, by COV and transitivity, $L_{\varphi}$ is dense in $I_{\varphi}$. This contradicts Theorem 7.3. If $\alpha_{1}=0$, a similar argument applies.

For $n \geq 3$ the answer is less clear-cut. Using the techniques of Section 5.2, for an arbitrary $I_{\alpha} \subset \mathbb{R}_{++}^{n}$ we shall construct an LDR decomposition which is not associated with any WPO, COV and IIA bargaining solution on the domain of all $n$-person compact bargaining problems.

Let $0 \neq \alpha \in \mathbb{R}_{+}^{n}$ and let $B$ be a basis of $H_{\alpha}$ (cf. Theorem 5.4). Take a fixed $b^{*} \in B$. Define the assignment $f: B \rightarrow \mathbb{R}$ by, for all $b \in B, f(b)=1$ if $b=b^{*}$ and $f(b)=0$ otherwise. We also write $f: H_{\alpha} \rightarrow \mathbb{R}$ for the induced additive functional.

Lemma 7.5 $D_{f} \neq I_{\alpha}$ and $D_{f}$ is a dense subset of $I_{\alpha}$.

Proof. Since $\rho^{-1}\left(b^{*}\right) \notin D_{f}$, it follows that $D_{f} \neq I_{\alpha}$. We show that $D_{f}$ is dense in $I_{\alpha}$. It suffices to show that $\operatorname{ker}(f):=\left\{x \in H_{\alpha} \mid f(x)=0\right\}$ is dense in $H_{\alpha}$. To this end, write the vector $\sqrt{2} b^{*} \in H_{\alpha}$ in terms of the basis $B$. So, we have a finite subset $E$ of $B$ such that

$$
\sqrt{2} b^{*}=\sum_{b \in E} q(b) b
$$

where all coefficients $q(b)$ are non-zero elements of $\mathbb{Q}$. By rewriting if necessary (namely in case that $b^{*} \in E$ ), we find that there is an irrational (and hence in particular non-zero) number $\lambda$ and a finite subset $F$ of $B$ that does not contain $b^{*}$ and rational coefficients $q(b)$ for each $b \in F$ such that

$$
\lambda b^{*}=\sum_{b \in F} q(b) b .
$$

Now take an arbitrary $x \in H_{\alpha}$. We show that $x$ is an element of the closure of $\operatorname{ker}(f)$. Write

$$
x=\sum_{b \in G} q(x, b) b
$$

where $G$ is a finite subset of $B$ and all $q(x, b)$ are non-zero rational numbers. If $G$ does not contain $b^{*}$, then $x \in \operatorname{ker}(f)$ and we are done. If $b^{*}$ is an element of $G$, then take a $q^{*} \in \mathbb{Q}$ such that the Euclidean distance between $q^{*} \lambda b^{*}$ and $b^{*}$ is smaller than $\varepsilon$. Then

$$
\sum_{b \in G \backslash b^{*}} q(x, b) b+q\left(x, b^{*}\right) \cdot q^{*} \lambda b^{*}=\sum_{b \in G \backslash b^{*}} q(x, b) b+q\left(x, b^{*}\right) \cdot q^{*} \cdot \sum_{b \in F} q(b) b
$$


is an element in $\operatorname{ker}(f)$ whose distance to $x$ is smaller than $q\left(x, b^{*}\right) \cdot \varepsilon$.

Combining Theorem 7.3 and Lemma 7.5 we obtain the following negative result.

Corollary 7.6 There does not exist a WPO, COV and IIA bargaining solution $\varphi: \mathcal{C} \rightarrow \mathbb{R}_{++}^{n}$ such that $I_{\varphi}=I_{\alpha}, L_{\varphi}=L_{f}, D_{\varphi}=D_{f}$ and $R_{\varphi}=R_{f}$.

Proof. By Lemma 7.5, $D_{f} \neq I_{\alpha}$ and $D_{f}$ is dense in $I_{\alpha}$. Therefore $L_{f}$ is dense in $I_{\alpha}$. The result now follows from Theorem 7.3.

On the positive side we mention that for any linear functional $f$ on $H_{\alpha}$ the associated WPO, COV and IIA bargaining solution on $\mathcal{F}$ does extend to $\mathcal{C}$, since the solution is obtained by maximizing a Nash product over $I_{\alpha}$. To obtain a full characterization of all WPO, COV and IIA solutions on the domain $\mathcal{C}$ remains an open problem.

\section{Further discussions}

In this final section we discuss (1) the independence of the axioms, (2) the cardinality of the set of WPO, COV and IIA solutions (3) related literature; (4) concludes.

\subsection{Independence of the axioms}

We show the independence of the axioms used in this paper.

Not WPO The bargaining solution $\varphi: \mathcal{F} \rightarrow \mathbb{R}_{++}^{n}$ defined by $\varphi(F):=F$ for all $F \in \mathcal{F}$ satisfies IIA, COV and CON, but not WPO.

Not IIA The bargaining solution WPO: $\mathcal{F} \rightarrow \mathbb{R}_{++}^{n}$ defined by, for all $F \in \mathcal{F}$,

$$
\mathrm{WPO}(F):=\{x \in F \mid x<y \text { implies that } y \notin F\}
$$

satisfies WPO, COV and CON, but not IIA.

Not COV The bargaining solution $\varphi: \mathcal{F} \rightarrow \mathbb{R}_{++}^{n}$ defined by, for all $F \in \mathcal{F}$,

$$
\varphi(F):=\left\{x \in F \mid \sum_{i} x_{i} \geq \sum_{i} y_{i} \text { for all } y \in F\right\}
$$

satisfies WPO, IIA and CON, but not COV.

Not CON Any bargaining solution discussed in subsection 5.2 falls in this category.

Note that the first three counterexamples can be extended to any domain $\mathcal{D}$ with $\mathcal{F} \subset \mathcal{D} \subset \mathcal{C}$. As for the continuity axiom, at least the bargaining solutions arising from technique I, as well 
as the ones arising from technique II for a linear functional, allow extension to such a domain. Hence, the independence of the axioms holds for any domain $\mathcal{D}$ with $\mathcal{F} \subset \mathcal{D} \subset \mathcal{C}$.

\subsection{The number of WPO, COV and IIA solutions}

One might think that, on the domain $\mathcal{F}$, every WPO, COV and IIA bargaining solution can be written as an iterated maximization of Nash products, similar to technique I in subsection 5.2. This, however, cannot be true, since the cardinality of the set of all WPO, COV and IIA bargaining solutions is, as we have seen, at least as large as the cardinality of $3^{B}$, where $B$ is a basis of $\mathbb{R}^{n}$ over $\mathbb{Q}$, while the set of all bargaining solutions that are an iterated maximization of Nash products has the cardinality of $\mathbb{R}^{n}$. Now, $B$ necessarily has the cardinality of $\mathbb{R}^{n}$, while, by the Cantor argument, $3^{B}$ has a higher cardinality than $B$.

Finally, it may still be true that bargaining solutions that are an iterated maximization or minimization of Nash products are indeed the only bargaining solutions that allow an extension to the class $\mathcal{C}$ of all compact bargaining solutions.

\subsection{Related literature}

Most of the results in the literature concerning Nash bargaining solutions on non-convex bargaining problems follow from our basic results (Theorem 3.3, Corollary 3.4, Theorems 4.1 and 4.2) and their applications in Sections 5, 6, and 7.

Kaneko [4] characterizes the symmetric Nash (multi-valued) bargaining solution on the domain of all compact $n$-person bargaining problems by the axioms PO, IIA, COV, SYM, and CON. A recent improvement of this result is obtained by $\mathrm{Xu}$ and Yoshihara [11], who are able to drop CON from this set of axioms. Both results follow from our Theorem 7.2, which, in turn, is a relatively straightforward consequence of the results in Sections 3 and 4.

Mariotti [5] characterizes the two-person symmetric Nash bargaining solution on the domain of finite bargaining problems by WPO, COV, SYM, and an axiom called 'Pareto monotonicity', which is implied by WPO and IIA. Hence, this characterization follows from Theorem 5.2. Theorem 4.3 in [5] about the symmetric two-person Nash bargaining solution on a larger domain follows from our Theorem 7.2. Observation 5.3.1 in [5] about the nonexistence of a two-person symmetric single-valued solution with the usual properties on a domain of compact connected problems is a particular consequence of Theorem 7.4.

Zhou [12] shows that, on the domain of compact $n$-person bargaining problems, any singlevalued solution satisfying COV, IIA, and strict individual rationality refines some Nash bar- 
gaining solution. As in Roth [9] it can be shown that these conditions imply WPO, and thus Zhou's result follows from our basic results in Section 3. Moreover, for the two-person case Theorem 7.4 shows that such single-valued selections are restricted to taking either the most left of the most right point within the Nash bargaining solution. Also for $n>2$ the choice of selection is most likely rather restricted, although it is not yet clear how exactly.

To our knowledge the papers mentioned so far are the ones that are most directly related to our work. Herrero [3] and Maschler et al. [7] consider the symmetric Nash bargaining solution on domains of compact bargaining problems from a more geometrical point of view, and complement it by a noncooperative game ([3]) or by a dynamic system ([7]). Conley and Wilkie [1] and Mariotti [6] study a different single-valued extension of the symmetric Nash bargaining solution to non-convex bargaining problems.

\subsection{Conclusion}

This paper has investigated the consequences of WPO, COV and IIA for bargaining solutions on the domain of finite bargaining problems and on larger domains. A few open problems remain, such as (1) an exact description of all solutions on the domain of compact bargaining problems for $n>2$, and (2) the necessity of the Axiom of Choice to derive (most of the) discontinuous solutions. Nevertheless, the results obtained are quite comprehensive and - to our knowledge - imply most of what is known in the literature.

\section{A Appendix: basic facts for the generalized indifference curve}

Throughout, let $\varphi: \mathcal{F} \rightarrow \mathbb{R}_{++}^{n}$ be a bargaining solution that satisfies WPO, IIA and COV. Clearly, by WPO, $x$ itself is an element of $I_{\varphi}(x)$ and each ray emanating from the origin into $\mathbb{R}_{++}^{n}$ intersects the generalized indifference curve at most once. We show that each ray also intersects this curve at least once.

Lemma A.1 Let $x, y \in \mathbb{R}_{++}^{n}$. Then $0<\lambda(x, y)<\infty$.

Proof. Because all coordinates of $x$ are strictly positive, by WPO and the non-emptiness of $\varphi(x, \lambda y)$ there exists a $\mu>0$ such that $\varphi(x, \lambda y)=\{x\}$ for all $0<\lambda<\mu$. Hence, $\lambda(x, y)>0$. Similarly, because all coordinates of $y$ are positive, there is a $\lambda>0$ such that $\varphi(x, \lambda y)=\{\lambda y\}$. Hence $\lambda(x, y)<\infty$. 
We need a few more facts about the generalized indifference curve. First, generalized indifference curves partition $\mathbb{R}_{++}^{n}$. Another way of saying this is that generalized indifference curves are the equivalence classes of an equivalence relation.

Lemma A.2 Let $x, y \in \mathbb{R}_{++}^{n}$ such that $y \in I_{\varphi}(x)$. Then $I_{\varphi}(x)=I_{\varphi}(y)$.

Proof. (a) Suppose that $z \in I_{\varphi}(y)$. We show that $z \in I_{\varphi}(x)$. Take $\lambda>0$.

(a1) Suppose $\lambda>1$. Then $\varphi(y, \sqrt{\lambda} z)=\{\sqrt{\lambda} z\}$ by Lemma 3.1. So, by COV, $\varphi(\sqrt{\lambda} y, \lambda z)=$ $\{\lambda z\}$. However, since $y \in I_{\varphi}(x), \varphi(x, \sqrt{\lambda} y)=\{\sqrt{\lambda} y\}$. Hence, $\varphi(x, \lambda z)=\{\lambda z\}$ by transitivity. (a2) Suppose $\lambda<1$. Then $\varphi(y, \sqrt{\lambda} z)=\{y\}$ by Lemma 3.1. So, by COV, $\varphi(\sqrt{\lambda} y, \lambda z)=$ $\{\sqrt{\lambda} y\}$. However, since $y \in I_{\varphi}(x), \varphi(x, \sqrt{\lambda} y)=\{x\}$. Hence, $\varphi(x, \lambda z)=\{x\}$ by transitivity.

(b) By (a1) and (a2), $\lambda(x, z)=1$ and hence $I_{\varphi}(y) \subset I_{\varphi}(x)$. We show that $x \in I_{\varphi}(y)$. Take $\lambda>0$.

(b1) Suppose $\lambda>1$. Then, since $y \in I_{\varphi}(x), \varphi\left(\frac{1}{\lambda} y, x\right)=\{x\}$. Hence, $\varphi(y, \lambda x)=\{\lambda x\}$ by COV.

(b2) Suppose $\lambda<1$. Then, since $y \in I_{\varphi}(x), \varphi\left(\frac{1}{\lambda} y, x\right)=\left\{\frac{1}{\lambda} y\right\}$. Hence, $\varphi(y, \lambda x)=\{y\}$ by COV.

By (b1) and (b2), $\lambda(y, x)=1$ and $x \in I_{\varphi}(y)$.

Lemma A.3 For every $x \in \mathbb{R}_{++}^{n}, I_{\varphi}(x)$ is closed.

Proof. Let $\left(y_{m}\right)_{m \in \mathbb{N}}$ be a sequence in $I_{\varphi}(x)$ that converges to $y$.

(a) Take $\lambda>1$. Since $y_{m} \rightarrow y$ there must be a $k \in \mathbb{N}$ such that $y_{k}<\lambda y$. Then there exists $\mu>1$ such that $\mu y_{k}<\lambda y$ also holds. Now, since $\varphi\left(x, \mu y_{k}\right)=\left\{\mu y_{k}\right\}$ and $\varphi\left(\mu y_{k}, \lambda y\right)=\{\lambda y\}$, we have $\varphi(x, \lambda y)=\{\lambda y\}$ by transitivity.

(b) Take $\lambda<1$. Since $y_{m} \rightarrow y$ there must be a $k \in \mathbb{N}$ such that $y_{k}>\lambda y$. Then there exists $\mu<1$ such that $\mu y_{k}>\lambda y$ also holds. Now, since $\varphi\left(x, \mu y_{k}\right)=\{x\}$ and $\varphi\left(\mu y_{k}, \lambda y\right)=\left\{\mu y_{k}\right\}$, we have $\varphi(x, \lambda y)=\{x\}$ by transitivity.

From (a) and (b) it follows that $\lambda(x, y)=1$ and hence $y \in I_{\varphi}(x)$.

Lemma A.4 Let $a \in \mathbb{R}_{++}^{n}$ and $y \in I_{\varphi}(x)$. Then ay $\in I_{\varphi}(a x)$.

Proof. Follows immediately from COV.

Lemma A.5 Let $a \in \mathbb{R}_{++}^{n}$. Suppose that ax $\in I_{\varphi}(x)$. Then $a^{q} x \in I_{\varphi}(x)$ for all $q \in \mathbb{Q}$. 
Proof. $\quad$ Take $a \in \mathbb{R}_{++}^{n}$ and $q \in \mathbb{Q}$. Suppose that $a x \in I_{\varphi}(x)$.

(a) First we show that $a^{k} x \in I_{\varphi}(x)$ for all $k \in \mathbb{N}$. Since $x, a x \in I_{\varphi}(x)$, we know that this is true for $k=0,1$. Suppose $a^{k} x \in I_{\varphi}(x)$ for $0 \leq k \leq l$. Since $I_{\varphi}(a x)=I_{\varphi}(x)$ by Lemma A.2, we have

$$
a^{l+1} x=a \cdot a^{l} x \in I_{\varphi}(a x)=I_{\varphi}(x)
$$

by Lemma A.4. Hence, $a^{k} x \in I(x)$ for all $k \in \mathbb{N}$.

(b) Next, take a $k \in \mathbb{N}, k \neq 0$. We show that $a^{\frac{1}{k}} x \in I_{\varphi}(x)$.

(b1) Take $\lambda>1$. Suppose that $x \in \varphi\left(x, \lambda a^{\frac{1}{k}} x\right)$. Then, because of COV, multiplication by $\lambda^{l} a^{\frac{l}{k}}$ shows that

$$
\lambda^{l} a^{\frac{l}{k}} x \in \varphi\left(\lambda^{l} a^{\frac{l}{k}} x, \lambda^{l+1} a^{\frac{l+1}{k}} x\right)
$$

for all $0 \leq l \leq k-1$. So, iterated application of transitivity yields that $x \in \varphi\left(x, \lambda^{k} a x\right)$. This contradicts the assumption that $a x \in I_{\varphi}(x)$. Hence, $\varphi\left(x, \lambda a^{\frac{1}{k}} x\right)=\left\{\lambda a^{\frac{1}{k}} x\right\}$.

(b2) Take $\lambda<1$. Suppose that $\lambda a^{\frac{1}{k}} x \in \varphi\left(x, \lambda a^{\frac{1}{k}} x\right)$. Then, because of COV, multiplication by $\lambda^{l} a^{\frac{l}{k}}$ shows that

$$
\lambda^{l+1} a^{\frac{l+1}{k}} x \in \varphi\left(\lambda^{l} a^{\frac{l}{k}} x, \lambda^{l+1} a^{\frac{l+1}{k}} x\right)
$$

for all $0 \leq l \leq k-1$. So, $\lambda^{k} a x \in \varphi\left(x, \lambda^{k} a x\right)$ by transitivity. This contradicts the assumption that $a x \in I_{\varphi}(x)$. Hence, $\varphi\left(x, \lambda a^{\frac{1}{k}} x\right)=\{x\}$.

From (b1) and (b2) it follows that $a^{\frac{1}{k}} x \in I_{\varphi}(x)$.

(c) Since $a x \in I_{\varphi}(x)$, we know that $x \in I_{\varphi}(a x)$ by Lemma A.2. Hence, by Lemma A.4, also $a^{-1} x \in I_{\varphi}\left(a^{-1} a x\right)=I_{\varphi}(x)$.

From (a), (b) and (c) it follows that $a^{q} x \in I_{\varphi}(x)$.

\section{References}

[1] Conley J, Wilkie S (1996) An extension of the Nash bargaining solution to nonconvex problems. Games and Economic Behavior 13:26-38

[2] Debreu G (1964) Continuity properties of Paretian utility. International Economic Review $5: 285-293$

[3] Herrero MJ (1989) The Nash program: non-convex bargaining problems. Journal of Economic Theory 49:266-277 
[4] Kaneko M (1980) An extension of the Nash bargaining problem and the Nash social welfare function. Theory and Decision 12:135-148

[5] Mariotti M (1998) Nash bargaining theory when the number of alternatives can be finite. Social Choice and Welfare 15:413-421

[6] Mariotti M (1998) Extending Nash's axioms to nonconvex problems. Games and Economic Behavior 22:377-383

[7] Maschler M, Owen G, Peleg B (1988) Paths leading to the Nash set. In: Roth AE (ed.) The Shapley value: essays in honor of Lloyd S. Shapley. Cambridge University Press, Cambridge UK, 321-330

[8] Nash JF (1950) The bargaining problem. Econometrica 18:155-162

[9] Roth AE (1977) Individual rationality and Nash's solution to the bargaining problem. Mathematics of Operations Research 2:64-65

[10] Shubik M (1982) Game theory in the social sciences: concepts and solutions. MIT Press, Cambridge Mass

[11] Xu Y, Yoshihara N (2004) Nonconvex bargaining problems. Games and Economic Behavior, forthcoming

[12] Zhou L (1996) The Nash bargaining theory with non-convex problems. Econometrica 65:681-685 